\title{
Branchial cleft cysts: a pictorial review
}

\author{
Antara Bagchi ${ }^{1 A, B, D, E, F}$, Priya Hira ${ }^{2 A, E}$, Kartik Mittal2 ${ }^{2 A, E}$, Aditi Priyamvara ${ }^{3 A, E}$, Amit K. Dey ${ }^{2 A, E}$ \\ ${ }^{1}$ Medical student, Indira Gandhi Government Medical College \& Hospital, Nagpur, India \\ ${ }^{2}$ Department of Radiology, Seth GS Medical College and KEM Hospital, Mumbai, India \\ 3Pandit Bhagwat Dayal Sharma University of Health Sciences, Rohtak, India
}

\section{Abstract}

Cystic masses of the neck may represent a wide variety of pathological conditions. Among these, branchial anomalies are the commonest paediatric congenital lesions of the head and neck. This article intends to review this condition under the same headings, as well as facilitate their diagnosis with the help of appropriate radiological findings of patients diagnosed with these anomalies. In order to make an accurate diagnosis, it is imperative to have an idea about the possible differentials, and the normal embryological development of the branchial arches, as well as their anomalies, in the form of sinuses, fistulae, and cysts, with their features and subtypes. The medical history of the patient and clinical manifestations helps in suspecting branchial cleft cysts; confirmatory imaging modalities include computed tomography, magnetic resonance imaging, ultrasonography, and fine-needle aspiration. The mainstay of management is usually by surgical excision. The location, clinical picture, and radiological correlation, along with a strong degree of suspicion for the condition, facilitates the diagnosis of this relatively common embryological anomaly.

Key words: branchial cleft cysts, branchial anomalies, CT, MRI, USG, FNAC.

\section{Introduction}

Embryology, although an intriguing subject matter, is complicated at the same time. It is fascinating how a singlecelled zygote transforms itself into a complete individual entity, capable of independent existence, by undergoing a complex series of differentiation and maturation. However, this process is such a sensitive and precisely controlled one that it is prone to be subjected to a myriad of derangements or irregularities; and here is where embryological anomalies or embryopathies come into the picture. These are developmental anomalies of a foetus or embryo, caused by environmental, genetic, or other unknown factors.

One of them is a branchial cleft cyst, which is one of the most frequently encountered congenital lesions of the head and neck among children [1].

\section{Embryology of the branchial apparatus}

During the embryological developmental process, the branchial or pharyngeal apparatus appears around the fourth week of gestation, in the region of the pharyngeal foregut, between the developing brain and heart. The apparatus consists of six pairs of arches, clefts, and pouches. Every arch contains a cartilaginous element, muscular component, a corresponding branch of the aortic arch, and a cranial nerve, and later give rise to various structures in the head and neck [2,3].

Occasionally, some defects can arise in the case of nonfusion or subinvolution of these arches, which leads to branchial cleft cysts (or lateral cervical cysts). These are epithelial cysts present on the lateral aspect of the neck, as remnants of the arches [4].

\section{Correspondence address:}

Dr. Amit K. Dey, MBBS, Department of Radiology, Seth GS Medical College and KEM Hospital, Acharya Donde Marg, Parel, Mumbai, India-400012,

phone: +91 8080995687, e-mail: amit5kem@gmail.com

\section{Authors' contribution:}

A Study design - B Data collection - C Statistical analysis - D Data interpretation - E Manuscript preparation · F Literature search · G Funds collection 
The first arch primarily produces the four muscles of mastication: the maxilla, mandible, malleus, and incus and Meckel's cartilage, and it is supplied by maxillary and mandibular branches of the trigeminal nerve, and the first aortic arch, which later persists as the maxillary artery. The second contributes to the formation of Reichert's cartilage, stapes, auricular and facial muscles, as well as platysma, and it is supplied by the facial nerve and second arch of aorta, which later gives origin to the stapedial artery. The third arch forms the stylopharyngeus, thymus, inferior aspect of the thyroid gland, and some parts of the hyoid bone, along with the internal and common carotid arteries. It is innervated by the glossopharyngeal nerve [4,5]. The fourth arch gives rise to the cricothyroid muscle, most of the intrinsic palatine muscles like the levator veli palatini (excluding the tensor veli palatini), and the epiglottic and thyroid cartilages. It is supplied by the superior laryngeal branch of the tenth cranial nerve and the fourth arch of aorta, whose right part gives rise to the subclavian artery and the left part to the aortic arch. The fifth arch has no contribution towards the development of any structure. The sixth arch is responsible for the formation of all the intrinsic laryngeal muscles (sans cricothyroid), cricoid cartilage, and the paired cartilages (arytenoid, corniculate, cuneiform). It is supplied by recurrent the laryngeal branch of the tenth nerve, and the sixth aortic arch, where the right part gives rise to the pulmonary artery and left sides gives rise to the pulmonary artery along with the ductus arteriosus.

The first three arches lead to the formation of structures above the larynx, while the fourth and fifth arch form structures below the larynx and contribute to the formation of tracheal structures. The nerves and arteries travel downwards to occupy their final positions in the thorax.

Eventually, the second arch overgrows downwards towards the fifth pharyngeal arch, which then leaves a depression, i.e. cervical sinus of His [5], which later obliterates on its own. However, on failure to obliterate, it can lead to the formation of branchial abnormalities like cysts (approx. 75\%), sinuses, or fistulae (25\%) [6,7]. Sinuses only possess a single internal opening, whereas fistulae have multiple internal as well as external openings.

\section{First branchial cleft cysts}

These are encountered infrequently and comprise only $7 \%$ of all recorded cases of branchial cysts, with around 200 cases documented in medical literature to date [8].

\section{Features}

On inspection, it looks like a round or oval cystic mass, which can be situated either within, superficial to, or deep to the parotid gland or along the external auditory canal. It is a possible differential for cystic lesions observed in the parotid or peri-parotid regions.
These cysts can be classified as:

- type 1: these are situated close to the external auditory meatus. In a large number of cases they are located inferior and posterior to the tragus. However, they can also be found in the parotid gland or at the angle of the mandible;

- type 2: these correspond to the submandibular gland and may be observed in the anterior triangle of the neck. These open into the external auditory canal.

The origins of type 1 and type 2 lesions are different. Type 1 aberrations are purely ectodermal, whereas type 2 comprise both ectodermal and mesodermal elements. The type 2 anomalies are greater in number and manifest as a replication of the membranous as well as cartilaginous elements of the external auditory canal. They mainly contain skin, adnexal structures, and cartilage; they may also be associated with the parotid gland [9].

\section{Clinical picture}

Recurrent parotid abscesses are commonly observed in patients of first branchial cleft cyst. These abscesses are indifferent towards antibiotics or incision and drainage. Occasionally, a sinus tract extending to the hyoid bone may be detected. The patient may complain of otorrhoea, in which case the cyst drains into the external auditory meatus. These anomalies may often mimic the clinical picture of parotid neoplasms and are likely alsoto be associated with facial nerve palsy because of their proximity to the seventh cranial nerve trunk [10]. As well as the paediatric population, adults can also be affected and are not immune to it $[11,12]$. On examination, they look like a fistula or a cystic mass, situated behind the pinna and concha.

\section{Management}

Treatment options solely include surgical excision $[11,12]$. The surgical method is unique for every case of this anomaly because it is imperative that the integrity and patency of the tract be maintained for complete excision. Dyes like methylene blue and gentian violet can be employed to facilitate identification of the tract. Moreover, the preservation of the facial nerve is yet another matter of paramount importance in such surgeries because the relation of the nerve to the cyst or track is variable and needs to be meticulously defined for careful and precise dissection in every case [13].

\section{Second branchial cleft cysts}

This variant of branchial cleft cysts makes up approximately $95 \%$ of all branchial anomalies. These can have several locations: along or adjacent to the anterior sternocleidomastoid border or at any point throughout the length of a second branchial fistula, extending from the skin of the lateral neck, between the external and internal carotid, and finally in the palatine tonsil. 

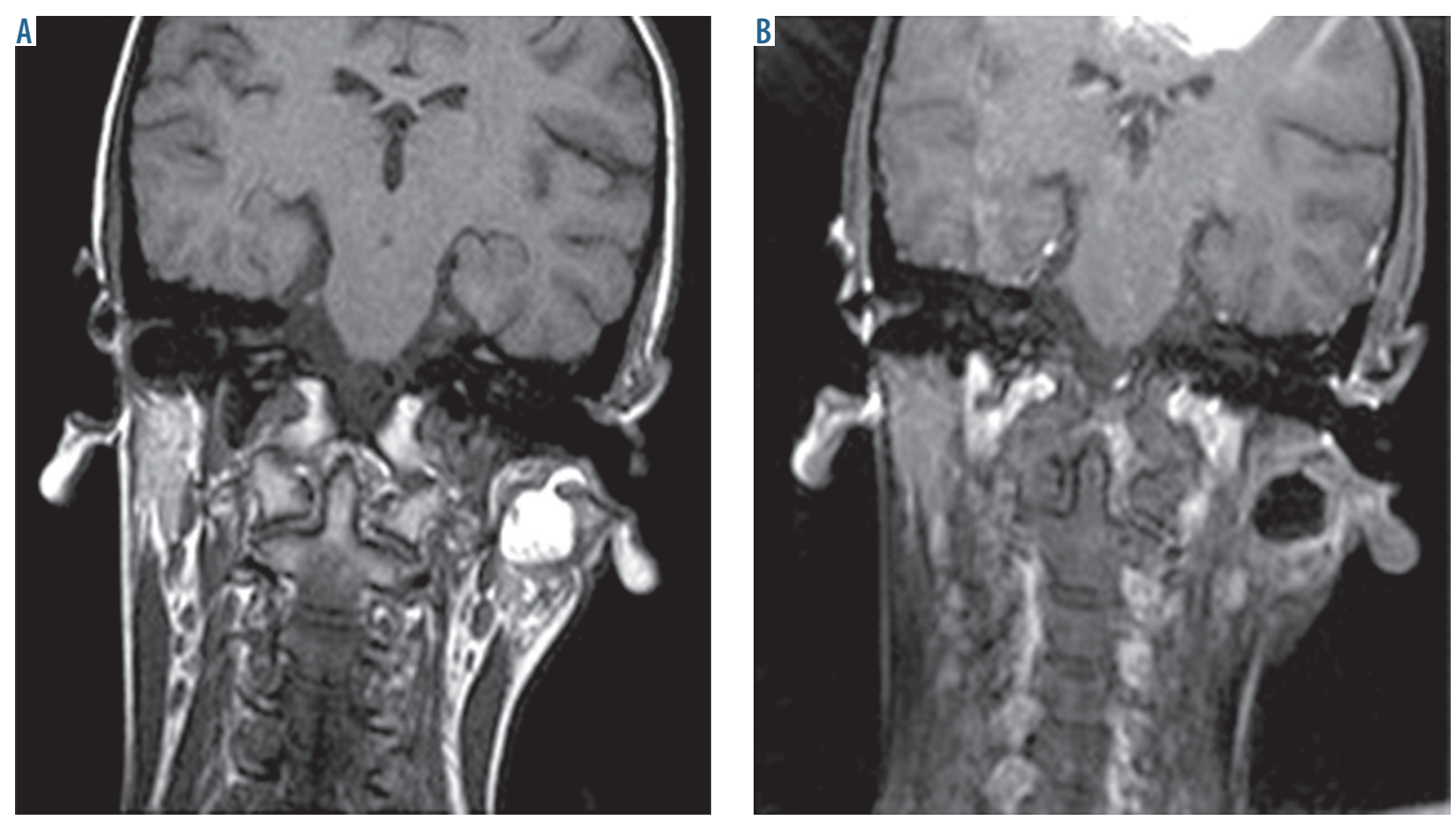

Figure 1. Normal and contrast-enhanced computed tomography images, taken in the coronal plane, revealing a well-defined hypodense mass

They are classified into four subtypes, as follows [14]:

- type 1: situated anterior to the sternocleidomastoid, just deep to the platysma,

- type 2: the most common variant of the four subtypes, found deep to the sternocleidomastoid, lateral to the carotid space,

- type 3: these extend medially between the bifurcation of internal and external carotid arteries up to the lateral pharyngeal wall,

- type 4: positioned in the pharyngeal mucosal space, medial to the carotid sheath.

\section{Features}

On gross examination, the physician may find internal septations or thick walls. Thickening of the walls can be a repercussion of an inflammatory process. In the case of fistula or sinus, the internal opening may be found in the region of the palatine tonsillar fossa. The external opening here is along the intersection of the middle and distal portions of the anterior border of the sternocleidomastoid. The cysts usually contain a turbid, yellowish fluid, which may include cholesterol crystals in their composition and can range from $1 \mathrm{~cm}$ to $10 \mathrm{~cm}$ in size $[7,10]$.

Histologically, the lining of these cyst walls is made of stratified squamous epithelium $[7,15,16]$.

\section{Clinical picture}

The cysts give the impression of painless, fluctuant masses, situated at the lateral aspect of the neck, proximal to the anteromedial border of the sternocleidomastoid, at the mandibular junction [15-17]. They gradually progress in size, and may become painful or tender with time if they are subsequently infected [15]. In the case of fistula, the ostium of the cyst would be discernible at birth, above the clavicle in the anterior aspect of the neck.

As per a case report regarding an oropharyngeal branchial cleft cyst, by Choo et al., the cystic mass was misdiagnosed as a mucocele. After its removal and consequent histological examination, it was found to be a branchial cleft cyst due to the presence of stratified squamous epithelial lining and lymphoid aggregation [18]. A recurrent inflammation in the region of the mandibular angle strongly indicates a second branchial cyst [19].

Diagnosis of the condition becomes relatively simple when the location is in its classic position. On USG, it looks like an anechoic mass or chiefly hypoechoic cystic mass, with faint internal debris and posterior enhancement. If it looks like a pseudo-solid heterogenous mass with internal debris and septa, the list of differentials would include lymphadenopathy, lipoma, nerve sheath tumour, inflammatory lesions, carotid body tumour, external laryngocele, and cystic hygroma. On a CT scan, the cyst usually appears as a well-circumscribed, non-enhancing mass of homogeneous low attenuation (Figures 1-4).

Occasionally, a curved rim of tissue, inclined in a way that is similar to pointing between the internal and external carotid arteries medially, also known as the "beak sign", can be observed on CT or MRI. It is pathognomonic of a type 3 second branchial cleft cyst.

\section{Management}

Since there is a possibility of secondary infection, the preferred management strategy is surgical excision [20]. 


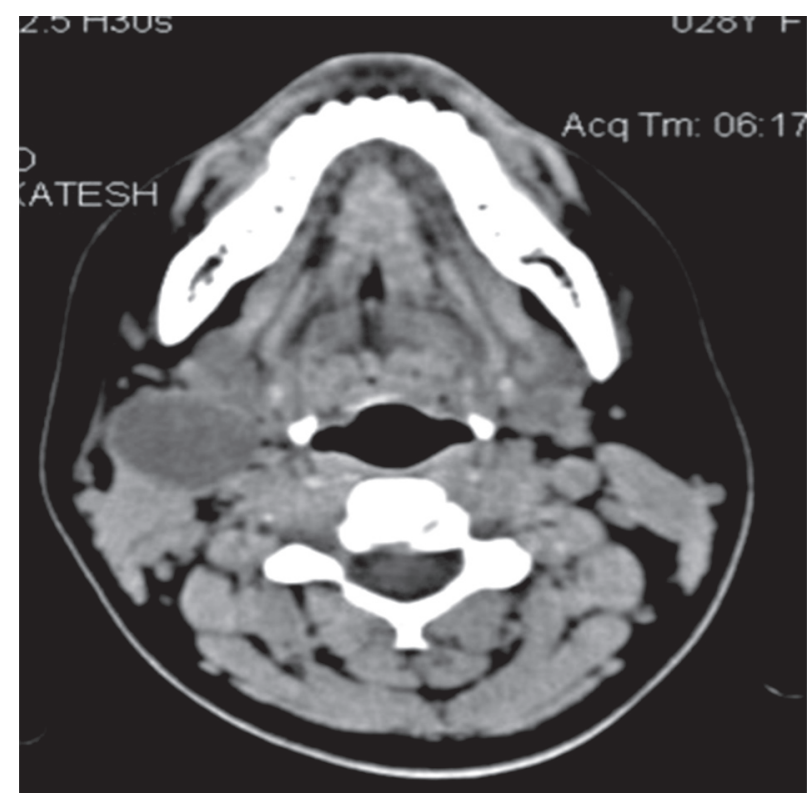

Figure 2. Contrast-enhanced computed tomography scan shows a cystic lesion in the right aspect of the neck, situated lateral to the carotid sheath and behind the submandibular gland, extending along the anterior border of the sternocleidomastoid muscle

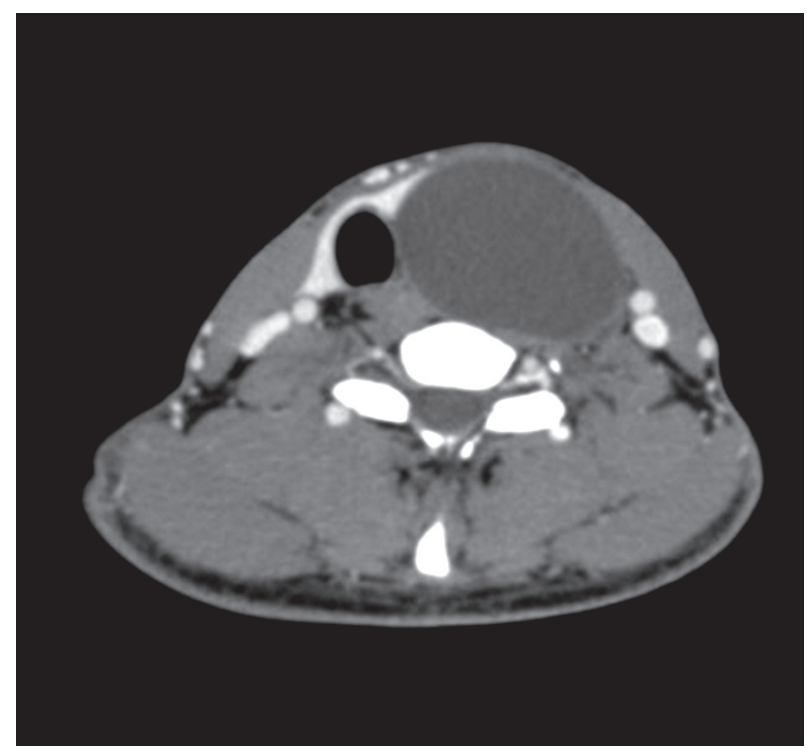

Figure 3. Contrast-enhanced computed tomography scan, taken in an axial plane at the level of the thyroid cartilage. A large, well-defined, nonenhancing water attenuation mass is evident, deep to the left sternocleidomastoid muscle, which dislodges the common carotid artery and internal jugular vein to the medial edge

It is normally delayed until the age of two to three years. In cases of isolated type 4 second branchial cleft cyst, an intraoral approach can be considered.

\section{Third branchial cleft cysts}

Third branchial cleft cysts are rarely encountered. However, they have been found to be the second most common congenital lesions of the posterior cervical area after thyroglossal cysts [21].

\section{Features}

They can be situated at any point in the tract that extends posterior to the carotid arteries and pierces the thyrohyoid membrane and enters the larynx, thus terminating on the lateral aspect of the pyriform sinus. The cysts are characteristically located deep to the sternocleidomastoid. It has been noted that these third branchial cleft cysts, when diagnosed in children, are found to be more commonly situated on the left side [4].

Anatomically, the third and fourth arch sinuses lie in close proximity to each other, and it therefore becomes a tedious task to distinguish between the two types simply by imaging modalities. To differentiate between the two, the position of superior laryngeal nerve with respect to the sinus tract must be considered. The third arch sinuses originate from the base of the pyriform sinus and proceed superior to the superior laryngeal nerve and hypoglossal nerve, but inferior to the glossopharyngeal nerve, and continue posteriorly to the carotid artery. The fourth arch sinuses arise from the apex of the pyriform sinus, continue inferior to the superior laryngeal nerve, and go down the tracheoesophageal groove. The main basis for making this distinction is by the correspondence of the superior laryngeal nerve to the sinus tract. Thus, the surgical route is recommended as the best for an accurate diagnosis [22].

\section{Clinical picture}

They are usually asymptomatic but can manifest clinically in the case of an additional infection. The prime diagnostic interventions are CT and MRI, in order to gauge the depth and extent of the branchial cleft cysts. On MRI, the contents and the wall of the cyst can be perceived as hypointense or even hyperintense to muscle in the presence of proteinaceous debris. On T2-weighted images, these are perceived as hyperintense lesions. In the case of an infection they can be confused with lymphadenopathy or an abscess due to an abnormal ring enhancement $[17,22]$.

\section{Management}

Imaging modalities are imperative for the diagnosis and management of these cysts. Definitive management for third branchial cleft cysts includes surgical excision. Visualisation of the pyriform sinus is necessary before surgery, and the favoured approach is along the sternocleidomastoid muscle.

\section{Fourth branchial cleft cysts}

\section{Features and clinical picture}

Fourth branchial cleft cysts are sparsely prevalent and sporadically observed cases. They comprise 1 to $4 \%$ of all the branchial cleft anomalies [23,24]. Like third BCCs, these are common on the left aspect. They are typically 

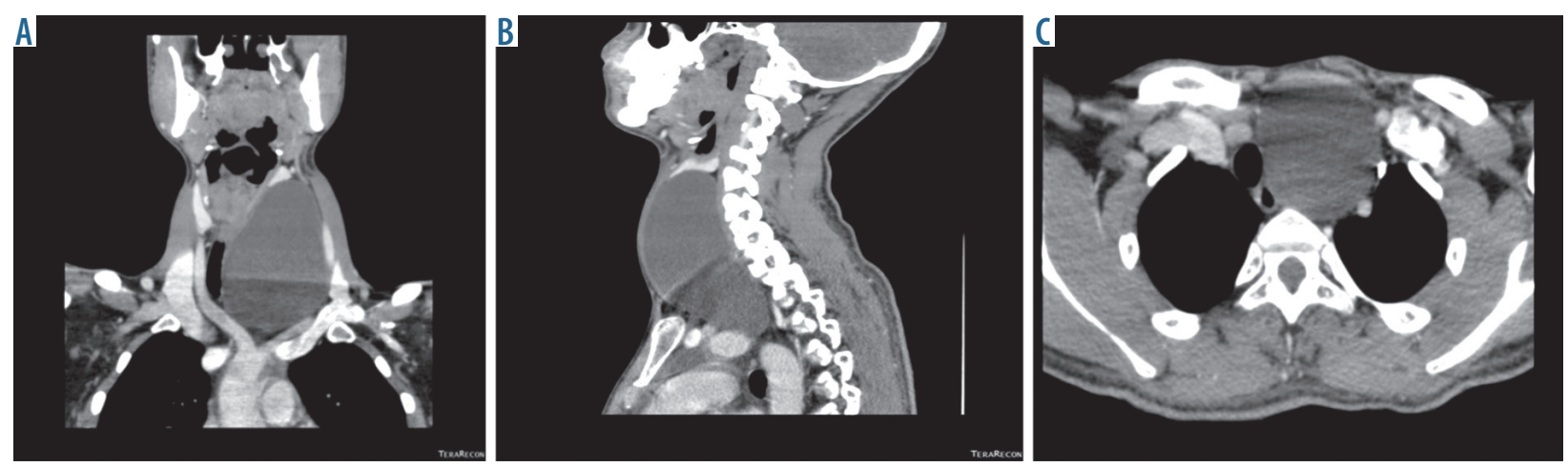

Figure 4. Contrast-enhanced computed tomography scan, taken at the level of the thyroid cartilage. A large, well-defined, non-enhancing water attenuation mass is noticeable at the axial, coronal, and sagittal planes, deep to the left sternocleidomastoid muscle

situated in the thyroid gland and mediastinum. These are present in early childhood, frequently after a recurrent abscess or a preceding thyroiditis (which can be superimposed, acute, and suppurative).

The sinus tract stretches from the apex of the pyriform sinus medial and inferior to the recurrent laryngeal nerve, loops superior to the hypoglossal nerve, parallels the course of the recurrent laryngeal nerve within the trachea-oesophageal groove, and wraps around the aortic arch on the left or subclavian artery on the right. It then runs superiorly, dorsal to the common carotid artery, loops around the hypoglossal nerve, and extends medially to the sternocleidomastoid muscle [25].

\section{Management}

Due to their rare occurrence, there are no concretely established procedures outlined for their management.
The definitive treatment is considered to be surgical excision combined with partial thyroidectomy [26].

\section{Conclusions}

This review article aims to elaborate the anatomical and radiological features of all the variants of branchial cleft cysts in order to help clinicians to identify and diagnose them accurately, and thus opt for the requisite management remedies appropriately. The location, clinical picture, and radiological correlation, along with a strong degree of suspicion for the condition, facilitates the diagnosis of this relatively common embryological anomaly.

\section{Conflict of interest}

The authors report no conflict of interest.
References

1. Goff CJ, Allred C, Glade RS. Current management of congenital branchial cleft cysts, sinuses, and fistulae. Curr Opin Otolaryngol Head Neck Surg 2012; 20: 533-539.

2. Koch BL. Cystic malformations of the neck in children. Pediatr Radiol 2005; 35: 463-477.

3. Al-Ghamdi S, Freedman A, Just N, et al. Fourth branchial cleft cyst. J Otolaryngol 1992; 21: 447-449.

4. Houck J. Excision of branchial cysts. Oper Tech Otolayngol Head Neck Surg 2005; 16: 213-222.

5. Benson MT, Dalen K, Mancuso A, et al. Congenital anomalies of the branchial apparatus: embryology and pathologic anatomy. Radiographics 1992; 12: 943-960.

6. Cote DN, Gianoli GJ. Fourth branchial cleft cysts. Otolaryngol Head Neck Surg 1996; 114: 95-97.

7. Deane SA, Telander RL. Surgery for thyroglossal duct and branchial cleft anomalies. Am J Surg 1978; 136: 348-353.

8. Aronsohn RS, Batsakis JG, Rice DH, et al. Anomalies of the first branchial cleft. Arch Otolaryngol 1976; 102: 737.
9. Finn DG, Buchalter IH, Sarti E, et al. First branchial cleft cysts: clinical update. Laryngoscope 1987; 97: 136-140.

10. Telander RL, Deane SA. Thyroglossal and branchial cleft cysts and sinuses. Surg Clin North Am 1977; 57: 779-791.

11. Work WP. Newer concepts of first branchial cleft defects. Laryngoscope 1972; 82: 1581-1593.

12. Work WP. Cysts and congenital lesions of the parotid gland. Otolaryngol Clin North Am 1977; 10: 339-343.

13. Quintanilla-Dieck L, Virgin F, Wootten C, et al. Surgical Approaches to First Branchial Cleft Anomaly Excision: A Case Series. Case Rep Otolaryngol 2016; 2016: 3902974.

14. Bailey $H$. Branchial cysts and other essays on surgical subjects in the facio-cervical region. Lewis \& Company, London 1929.

15. Som PM, Sacher M, Lanzieri CF, et al. Parenchymal cysts of the lower neck. Radiology 1985; 157: 399-406.

16. Som P. Cystic lesions of the neck. Postgrad Radiol 1987; 7: 211-236.

17. Miller MB, Rao VM, Tom BM. Cystic masses of the head and neck: pitfalls in CT and MR interpretation. AJR Am J Roentgenol 1992; 159: 601-607. 
18. Choo MJ, Kim YJ, Jin HR. A case of second branchial cleft cyst with oropharyngeal presentation. J Korean Med Sci 2002; 17: 564-565.

19. Harnsberger H. Handbook of head and neck imaging. $2^{\text {nd }}$ ed. Mosby - Yearbook, St. Louis 1995.

20. Telander RL, Filston HC. Review of head and neck lesions in infancy and childhood. Surg Clin North Am 1992; 72: 1429-1447.

21. Koeller KK, Alamo L, Adair CF, Smirniotopoulos JG. From the Archives of the AFIP: Congenital cystic masses of the neck: Radiologicpathologic correlation. Radiographics 1999; 19: 121-146.

22. Foxx SD, Smoker WR, Johnson MH, et al. Pediatric neck masses: Solid and cystic (a review). Radiologist (Baltimore) 1995; 2: 331-342.
23. Jeyakumar A, Hengerer AS. Various presentations of fourth branchial pouch anomalies. Ear, Nose Throat J 2004; 83: 640-644.

24. Nicollas R, Guelfucci B, Roman S, Triglia JM. Congenital cysts and fistulas of the neck. Int J Pediatr Otorhinolaryngol 2000; 55: 117-124.

25. Franciosi JP, Sell LL, Conley SF, Bolender DL. Pyriform sinus malformations: a cadaveric representation. J Pediatr Surg 2002; 37: 533-538.

26. Nicoucar K, Giger R, Pope HG, et al. Management of congenital fourth branchial arch anomalies: a review and analysis of published cases. J Pediatr Surg 2009; 44: 1432-1439. 